\title{
Diagnosis
}

\section{Review: screening tests are not as accurate as overnight polysomnography for the diagnosis of adult sleep apnoea}

Ross SD, Allen IE, Harrison KJ, et al. Systematic review of the literature regarding the diagnosis of sleep apnea. Evidence Report/Technology Assessment No 1. (Prepared by MetaWorks Inc. under contract no 290-97-0016.) AHCPR publication no. 99-E002. Rockville, MD:Agency for Health Care Policy and Research; February 1999.

QUESTION: How do the accuracies of diagnostic and screening tests (radiological imaging, laboratory assays, partial night polysomnography [PSG], daytime PSG, reduced channel PSG, and clinical signs and symptoms) compare with overnight sleep laboratory PSG for identifying sleep apnoea in adults?

\section{Data sources}

Studies were identified by searching Medline (1980 to November 1997) and Current Contents (1997) using the terms sleep apnoea syndrome; monitoring, physiologic; airway resistance; and index. Bibliographies were scanned.

\section{Study selection}

English, French, German, Spanish, and Italian language studies were selected if a diagnostic test or intervention was used to support a diagnosis of sleep apnoea and $\geq 10$ adults with any form of sleep apnoea were studied. Studies of patients with other potentially confounding diseases were excluded.

\section{Data extraction}

Data were extracted on study quality and description, patient and test characteristics, and results.

\section{Main results}

71 studies (7572 participants) compared overnight PSG (diagnostic standard) with portable monitoring devices (25 studies), reduced channel PSGs (3 studies), daytime PSGs (3 studies) partial night PSGs (4 studies), oximetry (12 studies), radiological tests (5 studies), clinical measures (17 studies), chemical assay ( 1 study), questionnaires (3 studies), and multivariate models (8 studies). Sensitivity and specificity for the tests vary (table).

\section{Conclusion}

Screening tests are not as accurate as overnight polysomnography for diagnosing sleep apnoea in adults.

Diagnostic and screening tests for sleep apnoea in adults using overnight laboratory polysomnography (PSG) as the diagnostic standard*

\begin{tabular}{lllll} 
Test & $\begin{array}{l}\text { Weighted } \\
\text { sensitivity }\end{array}$ & $\begin{array}{l}\text { Weighted } \\
\text { specificity }\end{array}$ & +LR & -LR \\
Oximetry & $87 \%$ & $65 \%$ & 2.5 & 0.2 \\
\hline Partial night PSG & $42 \%$ to $93 \% \dagger$ & $70 \%$ to $100 \% \dagger$ & - & - \\
\hline Daytime PSG & $66 \%$ to $100 \% \dagger$ & $50 \%$ to $100 \% \dagger$ & - & - \\
\hline Reduced-channel PSG & $82 \%$ to $94 \% \dagger$ & $82 \%$ to $100 \% \dagger$ & - & - \\
\hline FEF $_{50} /$ FIF $_{50}$ & $20 \%$ & $79 \%$ & 1.0 & 1.0 \\
\hline Sawtooth sign $^{\text {FEF }} /$ FIF $_{50}$ and sawtooth sign & $62 \%$ & $63 \%$ & 1.7 & 0.6 \\
\hline Global impression $_{\text {Prediction equations }}$ & $39 \%$ & $61 \%$ & 1.0 & 1.0 \\
\hline FEF & $59 \%$ & $66 \%$ & 1.7 & 0.6 \\
\hline
\end{tabular}

${ }^{\star} \mathrm{FEF}_{50} / \mathrm{FIF}_{50}=$ measure of extrathoracic airway obstruction. LRs defined in glossary and calculated from data in article. †Unweighted data.

\section{COMMENTARY}

The review by Ross and colleagues attempts to determine whether sleep studies without electrophysiological monitoring of sleep are clinically useful. It includes a serious methodological flaw: polysomnography is used as the diagnostic standard, but no evidence has shown that it is such. Polysomnography became accepted as the usual standard for diagnosing sleep apnoea in North America, but this conclusion was not evidence based. Studies that have attempted to determine whether electrophysiological monitoring adds to the accuracy of diagnosing sleep apnoea have found that it does not. ${ }^{1}$ Further, electrophysiological variables are not the best predictors of improvement with therapy. ${ }^{2}$

Despite this flaw, the review is useful because it shows the lack of agreement between diagnostic techniques and highlights the need for more carefully done studies. If the major clinical conclusion of the review is reduced to the statement that no severely symptomatic patient should have the diagnosis excluded on the basis of a negative limited sleep study, then I believe this is a reasonable, although not evidence based, statement. The finding of a pristine sleep and breathing pattern in all postures in a patient who is known to be asleep remains the best excluder of sleep apnoea. This belief has been reinforced by evidence from randomised controlled trials of continuous positive airway pressure (CPAP) therapy showing that large placebo responses can be obtained in patients with sleep apnoea. ${ }^{3}$ Thus, a response to a trial of CPAP cannot be used as evidence of disease, despite recent trends in this direction.

Neil Douglas, MD

Royal Infirmary Edinburgh, Scotland, UK

1 Douglas NJ, Thomas S, Jan MA. Lancet 1992;339:347-50.

2 Bennett LS, Lanoford BA, Stradling JR, et al. Am J Respir Crit Care Med 1998;158:778-86.

3 Engleman HM, Kingshott RN, Wraith PK, et al. Am J Respir Crit Care Med 1999;159:461-7.

4 Jenkinson C, Davies RJ, Mullins R, et al. Lancet 1999;353:2100-5.

Source of funding: Agency for Health Care Policy and Research

For correspondence Dr S D Ross,

MetaWorks, Inc., 470 Atlantic Avenue,

Boston, MA 02210

USA. Fax +1617368 3590. 